\title{
CLINICAL AND RADIOGRAPHIC EVALUATION OF IMMEDIATE IMPLANT WITH BONE GRAFT (HYPRO-OSS)
}

\author{
Mona Abd Elkader Abbas ${ }^{1}$, Walid Ahmed Ghanem ${ }^{2}$, Gihan El Desouky ${ }^{3}$, Yasser Mohamed El Gerby ${ }^{4}$
}

DOI: $10.21608 / d s u .2021 .23862 .1025$

Manuscript ID: DSU-2002-1025 (RI)

\section{KEYWORDS}

CBCT, Immediate placement, Xenograft bone grafts.

- E-mail address: dentistmonaabdelkader@gmail.com

1. Postgraduate Student in Oral and Maxillofacial Department, Faculty of Dentistry, Suez Canal University.

2. Professor Oral and Maxillofacial Surgery, Faculty of Dentistry, Suez Canal University.

3. Professor Oral Radiology, Faculty of Dentistry, Suez Canal University

4. Lecturer of Oral and Maxillofacial Surgery, Faculty of Dentistry, Suez Canal University.

\begin{abstract}
Introduction: Xenograft play role in osseointegration of immediate dental implant. Aim of the study: to evaluate the efficiency of xenograft (Hypro-oss) in osseointegration of immediate dental implant clinically and radiographically. Patients and methods: This study conducted on 8 patients range from 18-48 years old. 12 immediate dental implants divided into two equal groups; (Control Group): Atraumatic extraction of single rooted tooth or remaining root and immediate placement of dental implant, (Study Group): Atraumatic extraction of single rooted tooth or remaining root and immediate placement of dental implant and sealed with Hypro-Oss bone graft material. Clinical examinations done for all patients prior to surgery include examination of oral and para oral tissues and evaluation the intermaxillary space and the type of occlusion. Radiographic evaluation using CBCT done in the intervals 15 , 90,180 and 270 days postoperatively to measure the bone density of the formed bone between living bone and the implant surface. Result: All the patients showed no signs or symptoms of pain, tenderness, redness or inflammation of soft tissue around the inserted implant site. Normal healing process of the soft tissue and bone around the implant. All patients continued the follow up period. Radiographic evaluation showed significant higher bone density in study group than control group after 15, 90, 180 and 270 days. Conclusion: With the use of Hypro-Oss bone graft, the bone density around the implant showed increase in values.
\end{abstract}

\section{INTRODUCTION}

Immediate implant placement defined as the positioning of a dental implant immediately into fresh extraction socket after tooth extraction, has been considered a predictable and acceptable procedure for redressing missing teeth ${ }^{(1)}$.

Immediate placement of an endosseous implant after tooth extraction has various advantages. It sustains the horizontal and vertical dimensions of the bone, decreases treatment time, and preserves the implant in the same angulations as the previous natural tooth ${ }^{(2)}$.

The most significant aspect of any implant surgery regarding the successfulness of the procedure is implant stability and bone to implant contact (BIC) ${ }^{(3)}$. Moreover to achieve good oseo-integrated dental implant with a high degree of predictability, the implant shall be sterile, made of a highly bio-compatible material, e.g. titanium, inserted with 
an atraumatic surgical procedure that avoids overheating, placed with an initial stability and functionally loaded after healing period of 4-6 months ${ }^{(4)}$.

Although the clinical results of the implant are affected by factors including the body of the implant, the skill of the surgeon and the oral environment, the main factor for success is the primary stability at the implant placement. Initial stability can be increased with adequate bone quality then the osseointegration improved and the likelihood of the dental implant increased consequently ${ }^{(5)}$.

The basic stability associated with mechanical involvement of the implant with the surrounding bone after the implant is inserted; while bone regeneration and remodeling provide secondary osteointegration (biological stability) to the implant (6). The use of bone substitutes between titanium implant surface and the interior walls of the sockets will improve the primary stability of immediately placed implants, biological fixation and then the osseointegration process $^{(7)}$.

There are still challenges for the oral surgeon to determine the appropriate bone substitute around the inserted immediate implant to achieve a successful osseointegration and increase the success rate of dental implants.

\section{PATIENTS AND METHODS}

This study was approved by the ethical committee of Faculty of Dentistry Suez Canal University.

The study was conducted on twelve immediate dental implants fixed in eight patients. The implants divided into two equal groups: (Control Group): Atraumatic extraction of single rooted tooth or remaining root and immediate placement of dental implant into fresh extracted socket, while in (Study Group): Atraumatic extraction of single rooted tooth or remaining root and immediate placement of dental implant into fresh extracted socket and sealed with Hypro-Oss bone graft material. The patients selected from the outpatient clinic, oral and maxillofacial surgery department, Faculty of Dentistry, Suez Canal University. All patients were physically healthy and have good oral hygiene condition and given the necessary information about the procedure and they gave their consent to participate in a written informed consent.

Hypro-Oss is a natural bovine bone substitution material for permanent filling and reconstruction of bone defects. Each granule consists of 30\% AteloCollagen Type I and 70\% hydroxyapatite. HyproOss is produced by using patented atelopeptidation and lyophilization processes.

Preoperative phase: Detailed preoperative data collected from all patients through a printed questionnaire and discussion. Examination of oral and Para oral tissues done. Evaluation the intermaxillary space and the type of occlusion. CBCT radiographs taken for each patient to evaluate the absence of any pathology related to the tooth to be extracted, the length and width of the tooth for proper selection of the implant required for the surgery and Mesio-Distal and Bucco-Palatal Dimensions. Diagnostic casts construction done and all patients undergo scaling and root planning prior to the surgical procedure to ensure and preserve good oral health. All patients received strict oral hygiene instruction to preserve periodontal health in the form tooth brushing and oral rinses mouth wash with "Antiseptol""three times per day. Each patient was directed to administer oral prophylactic antibiotic Augmentin** twice daily one day before surgery.

\footnotetext{
* $0.12 \%$ chlorhexidinegluconate manufactured by kahira pharmaceutical company

** Amoxicillin 875mg. \&Clavulanic acid $125 \mathrm{mg}$ manufactured by Glaxowellcome.co
} 
Operative phase: The patients were directed to take antibiotic tablet one hour before surgery, patients were directed to thoroughly rinse (Antiseptol) with an antiseptic solution prior to surgical procedure. All the surgical procedures done under local anesthesia (Supraperiosteal injection-infiltration) using Ubistesin Forte 1:100.000 (Articane HCL $4 \%$ by ESPE). Local anesthesia administered to the patient just before surgery. The oral cavity purified using Betadine antiseptic solution and the patient draped using sterile towels reference to the standard technique of intraoral surgery.

A gingival incision made using bard parker No3 with blade \#15 exposing the tooth or remaining root to be extracted. The tooth was extracted atraumatically using the periotome. The periotome applied around the tooth to be extracted to cut and tear the periodontal ligaments. Appropriate forceps utilized with slight twisting movement and care should be taken to sustain the facial and palatal walls of the socket virtually intact. The socket thoroughly degranulated by careful curettage using small curette and proper irrigation with saline solution to remove any connective tissue tags or periodontal ligaments remnants.

The root diameter measured by using caliber to determine the diameter (mesio-distal and buccopalatal) and the depth gauge to determine the depth of the fresh extracted bony socket to select the appropriate implant. The implant should be as wide as permissible to allow maximum bone engagement with minimum thickness of facial and palatal walls not less than $1 \mathrm{~mm}$. The implant should extend 3 to $5 \mathrm{~mm}$ beyond the apex of the extracted root to achieve primary stability of the fixture.

(New biotech-type IS-II active) implant system was used.
The pilot hole drilled using a twist drill of $2 \mathrm{~mm}$ diameter to the planed depth, which extended to $3-5 \mathrm{~mm}$ apical to the depth of fresh extracted bony socket, sequential drills of gradual increased diameter were used under copious irrigation with saline until reach the suitable dimensions for the selected implant. As the implant is a self-tapping, it inserted to $2 / 3$ of its length under finger pressure, followed by slight tapping. The implant screwed into bone until the implant was below the alveolar bone crest by $1 \mathrm{~mm}$ (submerged implant). The cover screw screwed in place.

In-group 1 (control) no bone graft was placed. In-group 2 (study) the surrounding space, between the implant and the wall of the socket, filled with the bone graft (Hypro-oss). Interdental papillae mesial and distal to each implant sutured in an interrupted matters suture using resorbable suture material.

Postoperative care and follow up: Patients instructed to apply cold packs over the surgical area extra-orally $15 \mathrm{~min} / \mathrm{hr}$. for the first six hours postoperatively. Oral regimen of Augmentin1gm/ 12hr continued for five days post-operatively. Cataflam $50 \mathrm{mg}$ (Diclofenac potassium-Novartis pharma) tablet was given two times/day for five days post operatively. After the first twenty-four hours patients were instructed to use Antiseptol mouthwash 4 times per day.

Clinical Evaluation: Patients were recalled 24 hours the next day after surgery to evaluate clinically the presence of delayed bleeding or postoperative complications as redness, edema, swelling at surgical site, wound dehiscence, pain, discomfort or implant looseness. Regular checkups done on weekly bases during the first 15 days then 90,180 and 270 day postoperatively.

Radiographic evaluation: GALAXIS GALILEOS viewer software of Sirona cone-beam volumetric imaging gray scale value was used, intervals 
of 15 days, 90, 180 and 270 days postoperatively. The CBCT used to measure the bone density of the formed bone between living bone and the surface of an implant. Readings were recorded as following: Mesial and distal to every implant from coronal view at 3 points: the $1^{\text {st }}$ point $3 \mathrm{~mm}$, the $2^{\text {nd }}$ point
$6 \mathrm{~mm} \&$ the $3^{\text {rd }}$ point $9 \mathrm{~mm}$ away from implant crystal module respectively. Labial and palatal to every implant from cross sectional view at 3 points: the $1^{\text {st }}$ point $3 \mathrm{~mm}$, the $2^{\text {nd }}$ point $6 \mathrm{~mm} \&$ the $3^{\text {rd }}$ point $9 \mathrm{~mm}$ away from implant crystal module respectively.

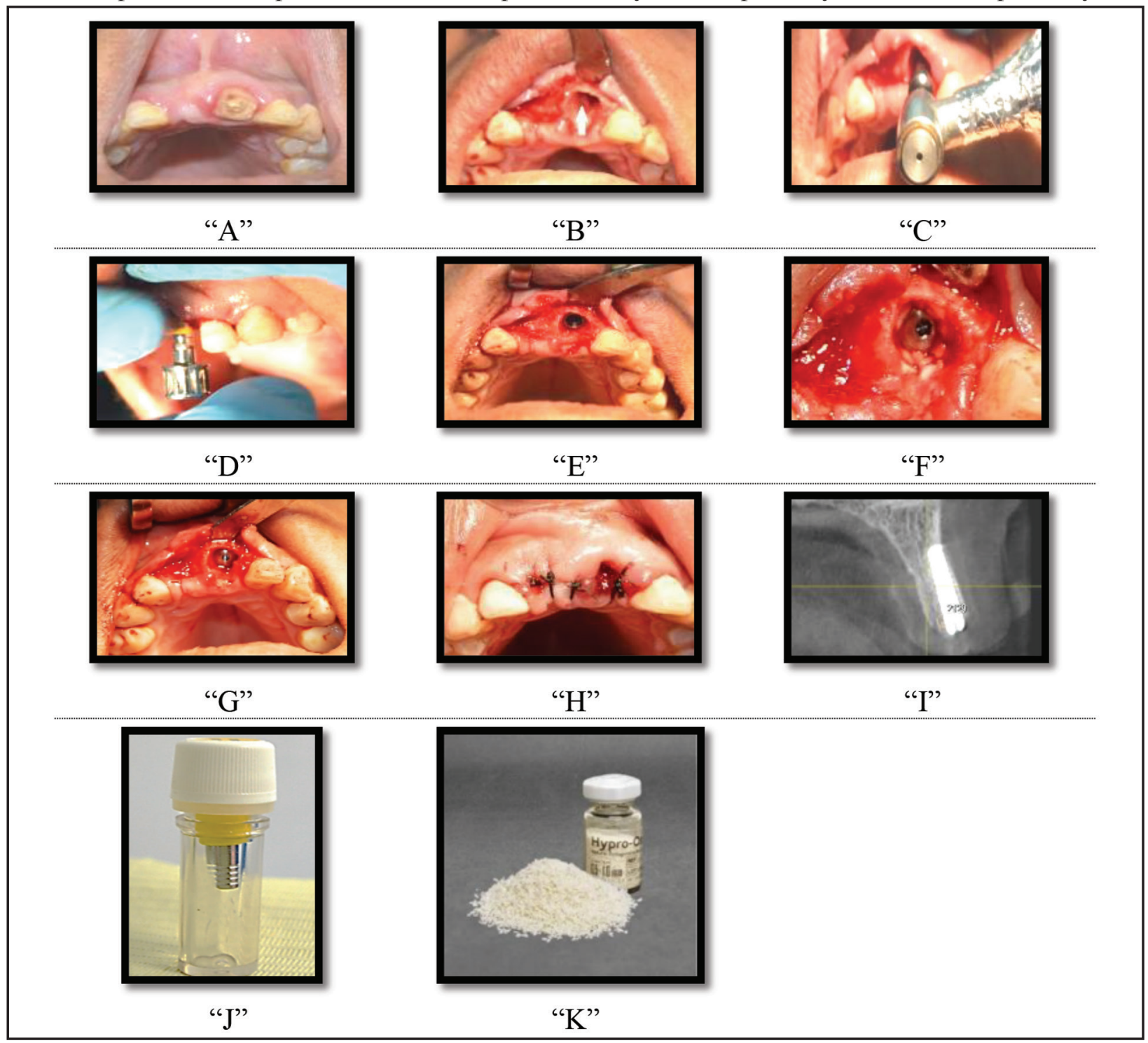

Fig. (1) Study group showing: (A) Showing non-restorable remaining root upper left central before extraction. (B) Showing fresh empty bony sockets after extraction and degranulation of the walls of the socket (White arrow). (C) Showing osteotomy preparation of extracted bony socket for immediate implant placement. (D) Showing-using screwdriver for immediate dental implant. (E) Showing the dental implant after insertion. (F) Showing cover screw of dental implant. (G) Showing the bone graft after packing into the gap space. (H) Showing the surgical field after suturing. (I) CBCT cross- Sectional showing bone density reading around inserted implant after 270 days. (J) Showing Neo Biotech implant. (K) Showing Hypro -Oss bone graft bottle granules. 


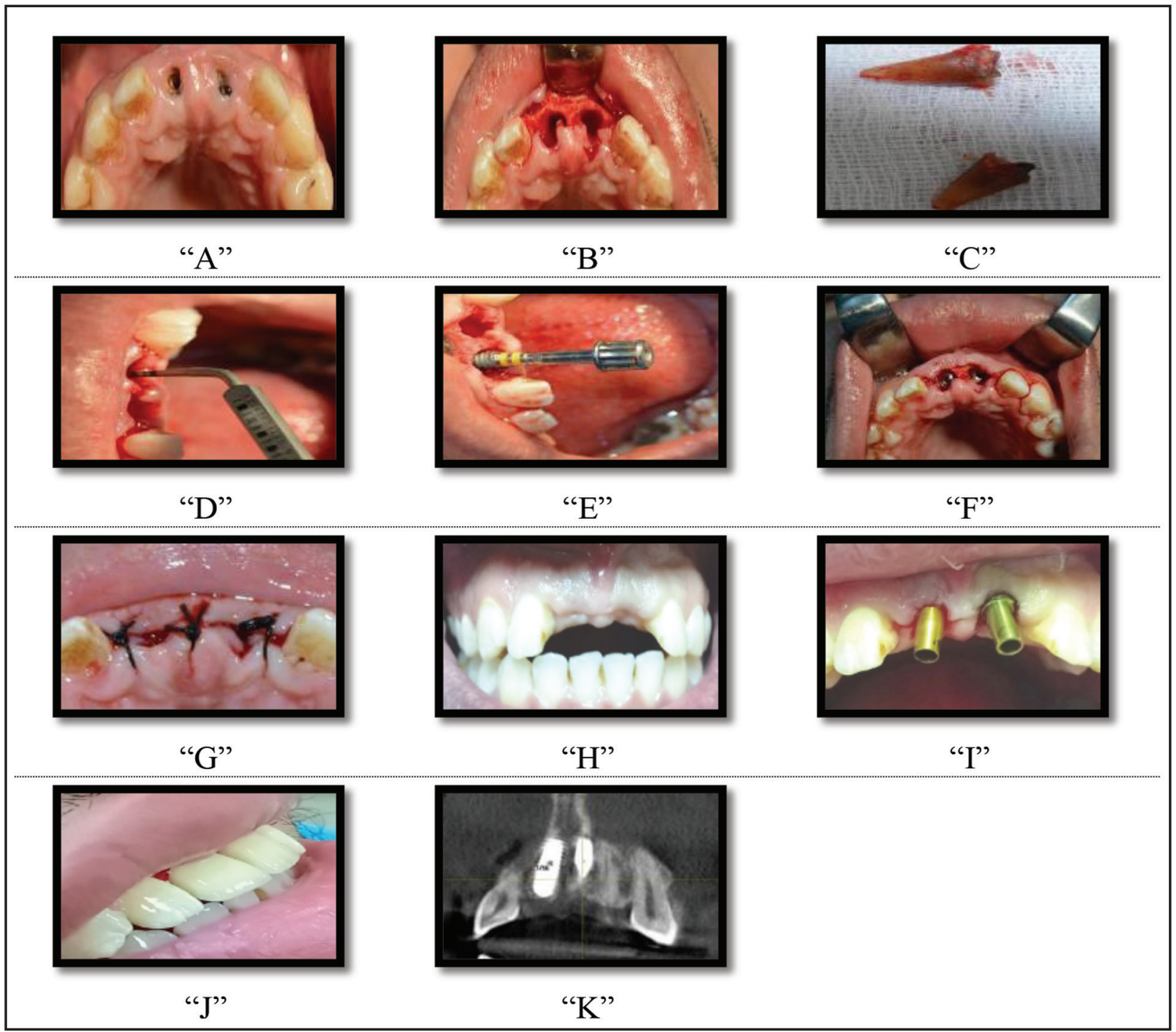

Fig. (2) Control group showing: (A) Showing non-restorable reaming root upper right-left central teeth and before extraction. (B) Showing fresh empty bony sockets after extraction and degranulation of the walls of the socket. (C) Showing the remaining teeth after extraction. (D) Showing the use of depth gauge for measuring the depth of the fresh extracted bony socket. (E) Showing screwdriver for dental implant. (F) Showing cover screw of dental implant. (G) Showing the surgical field after suturing. (H) Showing normal tissue healing over the implant site after 3 months. (I) Showing abutment connected to the fixture. (J) Showing the final restoration of implants that replacing missing bilateral upper two incisors. (K) CBCT crossSectional showing bone density reading around inserted implant after 270 days.

\section{Statistical analysis}

Data were analyzed using Statistical Program for Social Science (SPSS) version 20.0. Quantitative data were expressed as mean, \pm standard deviation (SD)., T-test and ANOVA were done. Independent
Samples (t-test) of significance was used when comparing between two means. A repeated analysis of variance (ANOVA) was used when comparing between more than two means. $\mathrm{P}$-value $<0.05$ was significant. 


\section{RESULTS}

Clinical evaluation: All the patients showed no signs or symptoms of pain, tenderness, redness or inflammation of soft tissue around the inserted implant site through the whole study. Normal healing process of the soft tissue and bone around the implant observed throughout the study. All patients continued the follow up period without any signs of infection, gingivitis or peri-implantitis. CBCT 15 days post operatively considered a base in comparison with other next intervals for bone density values in both groups.

Bone Density Measurements: In the control group; the first post operatively CBCT was done 15 days after implant insertion and considered base line for the following examinations done at 90, 180, and 270 days post operatively. The mean value stander deviation after 15 days postoperatively was (1114.00 \pm 222.98 ) while the mean value and stander deviation after 90 days was $(1509.67 \pm 94.72)$ while the bone density after 180 days was $(1685.83 \pm 101.72)$ and after 270 days was $(1903.17 \pm 117.28)$. Showing a high significant increase in the three records. P-value $<0.001^{*}$. In the study group, bone density measurements increased steadily throughout the study period showing statistical significant different mean value and stander deviation. After 15 days was $(1458.17 \pm 117.60)$ postoperatively and after 90 days was $(1754.50 \pm 75.81)$ while the mean value after 180 days reach to $(1958.50 \pm 84.42)$ and the mean value and stander deviation reach $(2295.67 \pm$ 115.21) after 270 days was showing high significant increase in the three records as was P-value $<0.001$. Table (1).

Table (1) Statistical analysis of bone density measurements around implants between control and study groups through the whole study intervals.

\begin{tabular}{|c|c|c|c|c|c|}
\hline & & Range & Mean \pm S. D & t. study & p. value \\
\hline \multirow{2}{*}{15 day } & Control group & $727-1321$ & $1114.00 \pm 222.98$ & \multirow{2}{*}{3.344} & \multirow{2}{*}{$0.007^{*}$} \\
\hline & Study group & $1296-1658$ & $1458.17 \pm 117.60$ & & \\
\hline \multirow{2}{*}{90 day } & Control group & $1347-1591$ & $1509.67 \pm 94.72$ & \multirow{2}{*}{4.943} & \multirow{2}{*}{$0.001 *$} \\
\hline & Study group & $1664-1844$ & $1754.50 \pm 75.81$ & & \\
\hline \multirow{2}{*}{180 day } & Control group & $1565-1798$ & $1685.83 \pm 101.72$ & \multirow{2}{*}{5.052} & \multirow{2}{*}{$0.001 *$} \\
\hline & Study group & $1856-2090$ & $1958.50 \pm 84.42$ & & \\
\hline \multirow{2}{*}{270 day } & Control group & $1704-2012$ & $1903.17 \pm 117.28$ & \multirow{2}{*}{5.848} & \multirow{2}{*}{$0.001 *$} \\
\hline & Study group & $2146-2459$ & $2295.67 \pm 115.21$ & & \\
\hline
\end{tabular}


The density of the bone in both groups is gradually growing. After 15 days postoperatively to 270 , the measurements of the bone density were higher in the study group. Gray scale readings of the bone density around the implant in study group showed significant increase more than control group according to statistical data analysis (t-study)

\section{DISCUSSION}

Cavallaro et $\mathbf{a l}^{(8)}$, concluded that when the treatment planned for extraction and potential immediate implant therapy the, teeth should be extracted in the most atraumatic manner possible. Forces should be applied with low velocity and high duration to minimize trauma to adjacent hard tissues and the section of multi-rooted teeth ought to be employed to prevent damage to facial plate and furcal bone, especially for molars.

This is in agreement with the surgical protocol that was applied in the current study and confirmed by the clinical evaluations: no signs or symptoms of pain, tenderness, redness, inflammation of soft tissue around the inserted implant site or implant looseness was detected.

Rojas-Vizcaya ${ }^{(9)}$, concluded that the position and angulation of the fixture should mainly be chosen primarily by determinants such as the need to achieve adequate primary stability and to be in line with an appropriately recognized restoratively driven plan. While Han and Jung ${ }^{(10)}$, mentioned that flaps should not be raised from the facial bone -if it can be avoided- because doing so severs the significant source of vascularization to the delicate facial plate, which might be as thin as less than $1 \mathrm{~mm}$ in the maxillary anterior teeth.

With the advent of immediate implant dentistry, implant fixtures are now regularly placed into fresh extraction sockets that, at their most coronal aspects, are sometimes much larger than the diameter of the implants being placed. There is usually somewhat of gap between the circumferential aspect of the immediate implant and the extraction socket wall. The dimension of the gap sometimes referred to as the jumping distance will vary depending on a number of factors, such as tooth type, the particular morphology of the extraction socket and the diameter of the implant being placed ${ }^{(11,12)}$.

A recent review of gap management concepts and techniques asserted that the immediate peri-implant gap consists of two dimensions: the horizontal defect width (between implant circumference and socket wall) and the vertical defect height [the distance between the most coronal aspect of the socket wall and the most coronal point of macroscopic contact between the fixture and the socket wall] ${ }^{(12)}$.

Ferrus et $\mathbf{a l}^{(13)}$, recommended that following implant placement, peri-implant gaps into which graft may be freely introduced (greater than $1 \mathrm{~mm}$ in dimension) should be grafted to prevent ridge width deficiency and promote greater bone-to-implant contact.

Autogenous bone grafting continues to be considered the gold standard for grafting and this has been studied by Ewers ${ }^{(14)}$, as he has used alternative graft material and followed-up the input/output statistic of implants to evaluate if this material (marine derived HA) results similar to the autogenous bone graft. Histomorphometric evaluations of graft materials show how much new bone is formed and if the graft material is resorbed. This study showed that the marine derived HA material ACA in a mixture with approximately $10 \%$ autogenous collector bone and blood or platelet rich plasma is able to enhance enough new bone in 6 months to allow implant osseointegration after 6 more months with a high implant survival rate. Allografts are the next best alternative at present; however, minor immunogenic rejection and risk of disease transmission are unresolved issues ${ }^{(15)}$. 
In the present study, xenogeneic bone graft was placed immediately in fresh post-extraction implant placement led to excellent clinical outcomes. The high success rate preservation of soft and hard tissue and general high patient satisfaction with the esthetic and functional outcomes show that this clinical procedure can be considered as safe, effective and predictable treatment option for the immediate rehabilitation of fresh post-extraction sockets $^{(16)}$.

Today it is well known that the positioning of implants during placement should be more palatally. During immediate implant placement, this creates the so called bucca 'gap' between the exposed implant surface and the bucca bone. This gap may then be filled with a variety of biomaterials capable of inducing bone formation ${ }^{(17)}$.

In the present study, we hypothesized that the use of a Xenograft containing atelo collagen would better serve the function of promoting more ideal bone regeneration. It was recently shown that the inclusion of atelo-collagen within the Xenograft provides numerous advantages when compared to Xenograft devoid of collagen. Atelo-collagen has shown to favour better growth factor adsorption, improve cellular attachment, promote faster cell proliferation and leads to greater osteoblast differentiation $^{(17)}$.

Xenograft (Hypro-Oss") that is composed of (atelo-collagen) with native osteoinductive elements and osteoconductive bovine Hydroxyapatite components, can be used as an efficient inductive bone grafting material for guided bone regeneration sinus augmentation.

This work can show that this Xenograft has various advantages due to the production process that preserves the organic elements- as bone growth factors that are responsible for accelerating the bone regeneration period and resulting in optimal bone quality and volume for optimizing implant placement. Good bone density was approved by high implant insertion torque and high volume of calcified tissue in histological slides. The clinical and radio graphical properties of the new bone are similar to natural type II bone. In addition to these advantages, a good handling during surgery, good healing and minimal post-surgical complications are concluded upon this case.

Antonin et $\mathbf{a l}^{(16)}$, conducted a study with the aim to ascertain the efficacy of phylogenic bone substitute hydroxyapatite in an augmented sinus. The process of graft healing, bone remodeling, and biomaterial replacement was examined. They concluded that after 15 months, the density of trabecula in grafted bone corresponded to cancellous bone was of good quality; however, the bone substitute was not completely resorbed during this period, indicating the affinity of the graft to induce more bone remodeling and increase in density. These results agree with the results of the current study as bone density curve is even increasing after 6 months.

Neugebauer et $\mathbf{a l}^{(18)}$, conducted a study to evaluate the effect of immediate loading of dental implants in conjunction with grafting procedures. The study was concluded that the local grafting did not disturb the course of osseointegration for immediate loaded implants if primary stability was reached. The regeneration of apical defect was uneventful and crestal defects required membrane fixation.

Regarding the previous study, immediate loading was avoided and delayed immediate loading was done showing a significant statistical improvement in both implant stability and bone density of the grafted group. All implants were loaded after six months after implant insertion. 


\section{CONCLUSION}

With the use of Hypro-Oss Bone graft the bone density around the implant showed increase in values especially after the implant was in function (post-loading of the implant).

\section{REFERENCE}

1. Tonetti MS, Cortellini P, Graziani F, Cairo F, Lang NP, Abundo R et al. Immediate versus delayed implant placement after anterior single tooth extraction: the timing randomized controlled clinical trial. J Clin Periodontol 2017;44: 215-224.

2. Terro W, Terro M. The effect of smoking and nonsmoking on bone healing (Osseo integrated phase) around lockingtaper dental implants. J Dent Impl 2015;5:12-15.

3. Bissinger O, Probst FA, Wolff K-D, Jeschke A, Weitz J, Deppe $\mathrm{H}$ et al. Comparative 3D micro-CT and 2D histomorphometry analysis of dental implant osseointegration in the maxilla of minipigs. J Clin Periodontol 2017; 44: 418-427.

4. Sanivarapu S, Dwarakanath CD, Ramesh AV. Immediate implant placement following tooth extraction: A clinical and radiological evaluation. Int J Oral Impl Clin Res 2010; 1:67-76

5. Fuh LJ, Huage CS, Chen KL, Shen MG. Variations in bone density at dental implant sites in different regions of the jaw bone - J Oral Rehab 2010; 37:346-351.

6. Barbisan A, Dias CS, Bavia PF, Sapata VM, César Neto JB, silva CO. Soft tissues changes after immediate and delayed single implant placement in esthetic area: a systematic review. J Oral Implantol 2015; 41:216-235.

7. Ignatius AA, Augat P, Ohnmacht M, Pokinsky JP, Kock HJ, Claes LE. A new bioresorbable polymer for screw augmentation in the osteosynthesis of osteoporotic cancellous bone: a biomechanical evaluation. J Biomed Mat Res 2001; 58:254-260.
8. Cavallaro J, Greenstein G, Tarnow DP. Clinical pearls for surgical implant dentistry, part 3. Dent Today 2010; 29:134-139.

9. Rojas-Vizcaya F. Biological aspects as a rule for single implant placement. Clin J Prosth 2013; 22:575-580.

10. Han JY, Jung GU. Labial and lingual-palatal bone thickness of maxillary and mandibular anteriors in human cadavers in Koreans. J Perio Impl Sci 2011; 41:60-66.

11. Clementini M. "Dimensional changes after immediate implant placement with or without simultaneous regenerative procedures: a systematic review and meta-analysis". J Clin Periodontol 2015; 42:666-677.

12. L Tettamanti. "post extractive implant: evaluation of the critical aspects". Oral Implantol (Rome) 2017; 10:119-128.

13. Ferrus J, Cecchinato D, Pjetursson EB. Factors influencing ridge alterations following immediate implant placement into extraction sockets. Clin Oral Impl Res 2010; 21:22-29.

14. Ewers R. Maxillary sinus grafting with marine algae derived bone forming material: a clinical report of long-term results. J Oral Maxillofac Surg 2005; 63:1712-1723.

15. Sakkas A, Wilde F, Heufelder M, Winter K, Schramm A. Autogenous bone grafts in oral implantology-is it still a "gold standard"? A consecutive review of 279 patients with 456 clinical procedures. Int J Implant Dent 2017; $3: 23$.

16. Antonin S, Michal C, Dana K, Ales K, Josef B, Dagmar V. The sinus lift with phycogenic bone substitute. Clin Oral Impl Res 2005; 16:342-348.

17. Fujioka-Kobayashi M, Schaller B, Saulacic N, Zhang Y, Miron RJ. Growth factor delivery of BMP9 using a novel natural bovine bone graft with integrated atelo-collagen type1: Biosynthesis, characterization, and cell behavior. J Biomed Mat Res 2017; 105:408-418.

18. Neugebauer J, Laze G, Perrotti V, Fischer JH, Khoury F, Pittelli, Zoeller JE. Experimental immediate loading of dental implants in conjunction with grafting procedures. J Biomed Mat Res 2009; 91:604-612. 